\title{
Dispersion of multi-walled carbon nanotubes in an aqueous medium by water-dispersible conjugated polymer nanoparticles $\dagger$
}

\author{
Beril Baykal, Vüsala Ibrahimova, Gizem Er, Erman Bengü* and Dönüs Tuncel*
}

Received 21st March 2010, Accepted 2nd August 2010

DOI: $10.1039 / \mathrm{c0cc00510j}$

Vertically aligned multi-walled carbon nanotubes (MWCNTs) synthesized by the alcohol catalytic CVD (ACCVD) technique are dispersed in water with the aid of water-dispersible conjugated polymer nanoparticles (CPNs). The interactions between CPNs and CNTs are studied with spectroscopy (UV-Vis, fluorescence and Raman) and electron microscopy techniques are used to confirm attachment of CPNs to the CNT sidewalls.

Carbon nanotubes (CNTs) are highly versatile materials with interesting mechanical and electrical properties and having wide application in the optoelectronics (e.g. LED and photovoltaic) and biomedical areas. ${ }^{1}$ However, the most important problem associated with CNTs is the solubility due to bundling caused by van der Waals interactions of carbon nanotube walls. Thus, it is important to solubilise carbon nanotubes using appropriate methods in order to expand their application areas.

Solubilisation can be realized by functionalization of the surface of the carbon nanotubes via covalent attachment of the suitable functional groups or by non-covalent interactions between the CNT surface and proper dispersants. ${ }^{2}$ Among many non-covalent solubilisation approaches, the use of conjugated polymers as dispersants is getting increasing attention because while this approach helps keep the good electrical and mechanical properties of CNTs untouched, unique electrical and optical properties can be created in CNTs via conjugated polymers $(\mathrm{CPs}){ }^{3}$ Thus, through functionalization a new composite material with controllable properties can be synthesized which can potentially find applications in many important areas such as LED, photovoltaic and biomedical applications. ${ }^{1}$

Depending on the conjugated polymer used as dispersant agent, the resultant $\mathrm{CP} / \mathrm{CNT}$ composite system can be made dispersible in organic solvents or in water. While conjugated polymers are not very efficient in solubilising CNTs in water, even incremental advances in this regard may be considered a significant step towards enabling widespread usage of CNTs, especially in biomedical applications, if good water solubility can be achieved through $\mathrm{CP} / \mathrm{CNT}$ composite systems.

To this end, we designed a new strategy to achieve good water dispersibility of carbon nanotubes and obtain $\mathrm{CP} / \mathrm{CNT}$ composite materials. In this approach, first a hydrophobic and non-water soluble conjugated polymer was converted into

Department of Chemistry and Institute of Materials Science and Nanotechnology, Bilkent University, Bilkent, Ankara, Turkey 06800. E-mail:dtuncel@fen.bilkent.edu.tr,bengu@fen.bilkent.edu.tr

$\dagger$ Electronic supplementary information (ESI) available: Experimental details, SEM image of pristine CNTs dispersion in water, TEM image of CPNs and EDX spectra of CPNs. See DOI: 10.1039/c0cc00510j

water dispersible nanoparticles and then the dispersion of carbon nanotubes in water with the aid of these conjugated polymer nanoparticles (CPNs) was achieved. The sizes of the CPNs were determined by dynamic light scattering (DLS) measurements and confirmed by scanning electron microscopy (SEM) and transmission electron microscopy (TEM). During TEM analysis of $\mathrm{CPN} / \mathrm{CNT}$ interaction energy-dispersive X-ray spectroscopy (EDX) was also used to confirm the presence of bromine in the polymer nanoparticles. Further characterisation to provide clues regarding the interaction between CPNs and CNTs was done by spectroscopic techniques (UV-Vis, fluorescence and Raman).

Poly[9,9-bis-(6'-bromohexylfluorene-2,7-diyl)-co-(benzene-1,4diyl)] (PFB-B) is synthesised by the Suzuki coupling reaction of monomer $1^{4}$ and phenylenediboronic acid following the reaction in Scheme 1.

PFB-B is characterized by spectroscopic methods $\left({ }^{1} \mathrm{H}-\mathrm{NMR}\right.$, UV-Vis, and fluorescence) and its molecular weight is determined by gel permeation chromatography (GPC). Its number average molecular weight is calculated as $1.4 \times 10^{4} \mathrm{Da}$ with polydispersity index of 2.3 .

We prepared conjugated polymer nanoparticles in two different sizes using the re-precipitation method. ${ }^{5}$ This method involves the dissolution of the polymer in a good solvent (e.g. THF) and injecting the polymer solution in a poor solvent (e.g. water) for the polymer and subsequent rapid stirring of the dispersion via ultrasonication. After the removal of the good solvent, nearly spherical nanoparticles are obtained. The size of the nanoparticles can be tuned by varying the concentration of the polymer and the volume of the poor solvent used. The average sizes of the nanoparticles were determined first by DLS as $\sim 70 \mathrm{~nm}$ and $\sim 40 \mathrm{~nm}$ average sizes as shown in Fig. 1.

After the synthesis and characterization of nanoparticles, we set out to investigate their interactions with CNTs. For this purpose we used vertically aligned multi-walled $\mathrm{CNTs}^{6}$ grown in our laboratory using the ACCVD technique. The reason for using vertically aligned CNTs was that because of the growth technique utilized the CNTs produced have a very tight diameter and length distribution. ${ }^{7}$ The CNTs synthesized using this technique have a very tight diameter

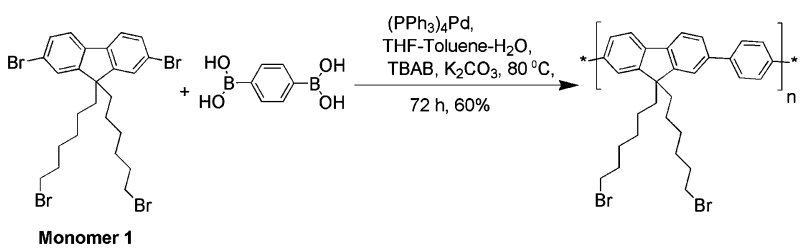

Scheme 1 Synthesis of poly[9,9-bis-(6'-bromohexylfluorene-2,7-diyl)alt co-(benzene-1,4-diyl)] (PFB-B). 

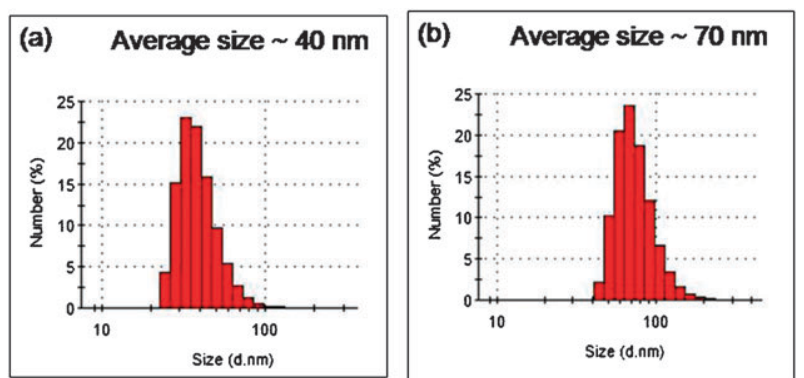

Fig. 1 Histograms for $40 \mathrm{~nm}$ (a) and $70 \mathrm{~nm}$ (b) CPNs measured by DLS.

and length distribution, and also don't require further purification. ${ }^{7}$ Vertically aligned CNTs grown on Si (100) substrates $\left(10 \times 10 \mathrm{~mm}\right.$ with a CNT density of $\left.1 \times 10^{10} / \mathrm{cm}^{2}\right)$ were then immersed in a vial with $\mathrm{CPN}-$ water dispersion. The contents of the vial were sonicated for about an hour. As a control, CNTs were also sonicated in a vial only with water.

As shown in Fig. 2a, upon introduction of CNTs the colour of the $\mathrm{CPN}$-water dispersion in the vial turns greyish indicating good dispersion. Good dispersion can be achieved with a minimum mass ratio of 0.2 for $\mathrm{CPN}$ to $\mathrm{CNT}$ demonstrating that $\mathrm{CPN}$ is a remarkably efficient dispersant for CNTs in water. The highest CNT concentration of stable dispersions is found to be around $0.25 \mathrm{mg} \mathrm{mL}^{-1}$ using $0.2: 1$ polymer to CNT mass ratio. Although some precipitation can occur after $12 \mathrm{~h}$, dispersions could be restored after a few minutes of sonication. We also noticed that CPNs with average size of $40 \mathrm{~nm}$ are better dispersants than the CPNs with larger average size; while we are further exploring this aspect, our initial findings indicate an interplay between CNT radius and $\mathrm{CPN}$ size.

The interaction between CPNs and CNTs was first investigated by UV-Vis and fluorescence spectroscopies. Fig. 3a shows the UV-Vis spectra of 40 and $70 \mathrm{~nm}$-sized CPNs in the presence and absence of CNTs in water. Absorption spectra did not indicate a significant change suggesting that the electronic structure of the nanoparticles was not altered upon complexation with CNTs. CPNs in water exhibit a strong fluorescence emission at $421 \mathrm{~nm}$. After dispersing the CNTs using 40 and $70 \mathrm{~nm}$-sized CPN-water dispersions, some quenching in the fluorescent emission of the dispersions was observed; $25 \%$ and $10 \%$, respectively. This is probably due to energy transfer (Fig. 3b) which seems to be more efficient with smaller CPNs $(40 \mathrm{~nm})$ than the larger CPNs $(70 \mathrm{~nm})$ suggesting the interaction between $40 \mathrm{~nm}$-sized CPNs and CNTs to be relatively stronger.

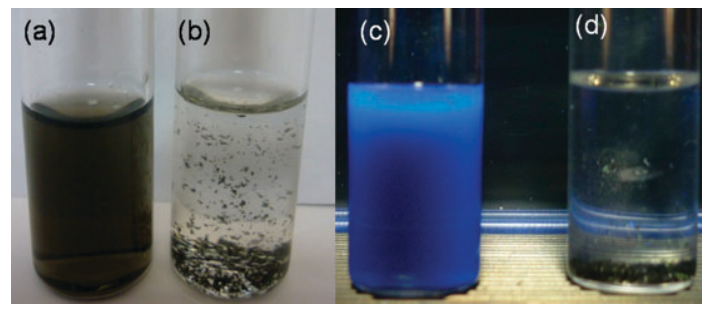

Fig. 2 Images of CPN (40 nm-sized)-CNT with 0.2:1 CPN to CNT mass ratio (a, c) and CNT-water dispersion (b, d) under ambient $(\mathrm{a}, \mathrm{b})$ and UV-light irradiation (c, d).

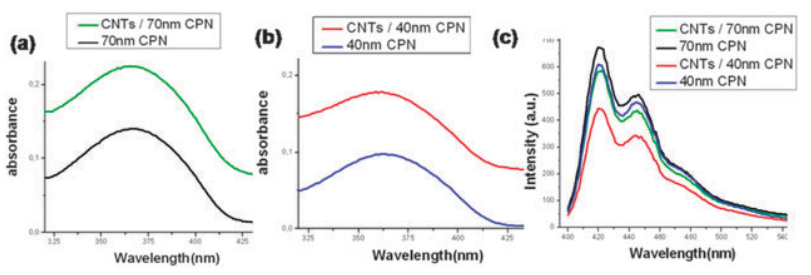

Fig. 3 The UV-Vis absorption (a, b) and emission spectra (c) of 40 and $70 \mathrm{~nm}$-sized CPNs in water and CPN-CNT dispersions.

Raman spectroscopy was also used to examine the interaction between CNTs and CPNs. The first-order Raman spectra from pristine CNTs dispersed in water and CPN-CNT mixtures are given in Fig. 4 and related numerical data are provided in Table 1. The CNTs dispersed in water show all of the expected first-order structures, namely D, G, and $\mathrm{D}^{\prime}$ bands. The origins for the first-order bands in CNT Raman spectra have been reviewed in detail recently. ${ }^{8}$ The intensity ratio of the $G$ band versus the $D$ band, $I_{G} / I_{D}$ can provide important clues regarding the defectiveness of CNTs, as the intensity of the D band is generally considered to be directly proportional to the defect density in the hexagonal carbon network of CNTs. ${ }^{9}$ In our study, we found that CPN-CNT mixtures indicated markedly lower $\mathrm{I}_{\mathrm{G}} / \mathrm{I}_{\mathrm{D}}$ ratios than $\mathrm{CNTs}$ dispersed in water, as shown in Table 1. A similar behaviour has been reported by Patole et al. ${ }^{10}$ for polystyrene attached CNTs where saturation of the dangling bonds in the hexagonal network has been offered as an explanation. Lower D band intensities and the disappearance of the $\mathrm{D}^{\prime}$ band for CPN-CNT mixtures as shown in Fig. 4 also suggest that the CPN/CNT interaction induced changes in the CNTs surface. We have also observed a small shift of the $G$ band to higher frequency for $\mathrm{CPN}-\mathrm{CNT}$ mixtures, which is also consistent with the interaction of polymer with CNT surface.

We have investigated the interaction between CPNs and CNTs using electron microscopy techniques, as well. SEM images in Fig. 5a and b clearly show CPN covered CNTs for both sizes of CPN-CNT dispersions. These images also show that the majority of CNTs are de-bundled, while SEM images from CNTs dispersed in water show bundled CNT layers (See ESI, Fig. S3). Furthermore, the dispersion of CNTs and

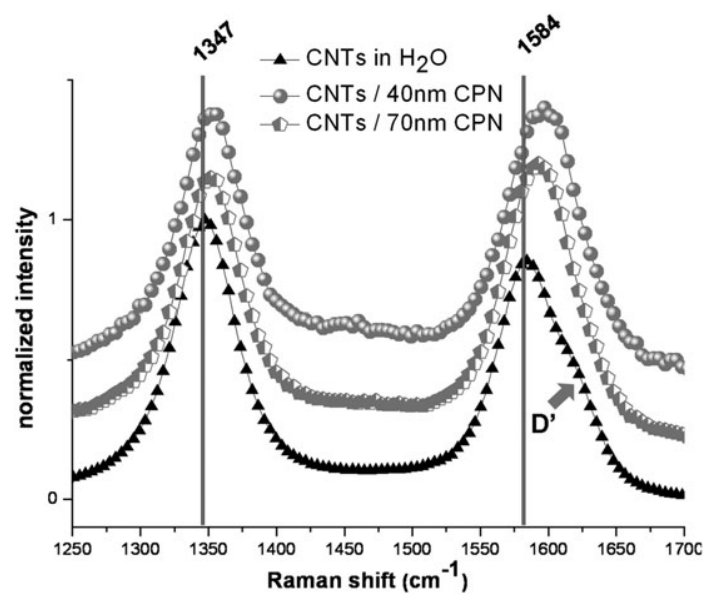

Fig. 4 The first-order Raman spectra from pristine CNTs dispersed in water and $\mathrm{CPN}-$ water dispersions. 
Table 1 Numerical data regarding Raman spectra analysis

\begin{tabular}{lllll}
\hline & D peak position $/ \mathrm{cm}^{-1}$ & $\mathrm{G}$ peak position $/ \mathrm{cm}^{-1}$ & $\Delta(\mathrm{G}-\mathrm{D}) / \mathrm{cm}^{-1}$ & $\mathrm{I}_{\mathrm{G}} / \mathrm{I}_{\mathrm{D}}$ \\
\hline $\mathrm{A}$ & 1347 & 1584 & 237 & 0.7 \\
$\mathrm{~B}$ & 1352 & 1593 & 241 & 0.8 \\
$\mathrm{C}$ & 1356 & 1597 & 241 & 1.0
\end{tabular}

A: Pristine CNT in water; B: CNTs/70 nm CPNs; C: CNTs/40 nm CPNs.

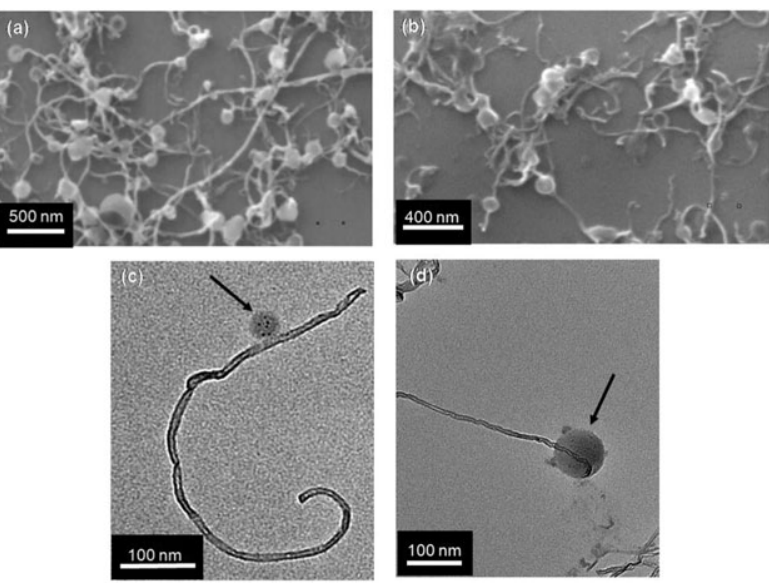

Fig. $540 \mathrm{~nm}$ (a) and $70 \mathrm{~nm}$ (b) CPNs attached to CNTs imaged by SEM; $40 \mathrm{~nm}$ (c) and $70 \mathrm{~nm}$ (d) CPNs by TEM.

the attachment of CPNs on nanotube surfaces were examined by TEM, as shown in Fig. 5c and d. EDX analysis clearly revealing the presence of bromine in nanoparticles attached on CNTs confirms CNT-CPN interaction (See ESI, Fig. S4). Overall, our data suggest an adequately strong and stable molecular interaction between the CPNs and CNT walls, most likely $\pi-\pi$ stacking interactions between the polymer aromatic backbones and carbon nanotube side-walls as well as due to the hydrophobic effect. We are continuing our efforts to provide more detail regarding this interaction using techniques such as advanced electron microscopy and first principles density functional theory calculations. ${ }^{11}$

In conclusion, we demonstrated that CNTs can be debundled and then dispersed in aqueous media in the presence of non-ionic conjugated polymer nanoparticles. Data gathered from spectroscopic and microscopic techniques evidently confirm the interaction of CPNs with CNTs. The optical properties of CPNs are not significantly altered upon complex formation indicating further that the interactions between CPNs and CNTs are non-covalent interactions. This feature can be advantageous for many applications such as light emitting diodes, bioimaging and biosensing. ${ }^{12}$

Support by EU-UNAM-REGPOT Grant No. 203953 and TUBITAK 109T026 is gratefully acknowledged. We also acknowledge the use of TEM facilities of Anadolu University under the supervision of Prof. Servet Turan and Pinar Kaya.

\section{Notes and references}

1 (a) M. Bansal, R. Srivastava, C. Lal, M. N. Kamalasananb and L. S. Tanwara, Nanoscale, 2009, 1, 317; (b) M. in het Panhuis, J. Mater. Chem., 2006, 16, 3598; (c) D. Tasis, N. Tagmatarchis, V. Georgakilas and M. Prato, Chem.-Eur. J., 2003, 9, 4000; (d) H.-C. Wu, X. Chang, L. Liu, F. Zhaoa and Y. Zhao, J. Mater. Chem., 2010, 20, 1036; (e) Carbon Nanotubes and Related Structures, ed. D. Guldi and N. Martin, Wiley-VCH, 2010.

2 (a) T. Palacin, H. L. Khanh, B. Jouselme, P. Jegou, A. Filoramo, C. Ehli, D. M. Guldi and S. Campidelli, J. Am. Chem. Soc., 2009, 131, 15394; (b) W. Z. Yuan, H. Zhao, X. Y. Shen, F. Mahtab, J. W. Y. Lam, J. Z. Sun and B. Z. Tang, Macromolecules, 2009, 42, 9400; (c) N. A. Kumar, S. H. Kim, B. G. Cho, K. T. Lim and Y. T. Jeong, Colloid Polym. Sci., 2009, 287, 97; (d) S. Park, H.-S. Yang, D. Kim, K. Job and S. Jon, Chem. Commun., 2008, 2876; (e) N. Wong, S. Kam, M. O'Connell, J. A. Wisdom and H. Da, Proc. Natl. Acad. Sci. U. S. A., 2005, 102, 11600; $(f)$ L. Lacerda, S. Faffa, M. Prato, A. Bianco and K. Kostarelos, Nanotoday, 2007, 2, 38.

3 (a) Y. K. Kang, O. Lee, P. Deria, S. H. Kim, T. Park, D. A. Bonnell, J. G. Saven and M. J. Therien, Nano Lett., 2009, 9, 1414; (b) J. Chen, H. Liu, W. A. Weimer, M. D. Halls, D. H. Waldeck and G. C. Walker, J. Am. Chem. Soc., 2002, 124, 9034; (c) J. Zou, S. I. Khondaker, Q. Huo and L. Zhai, Adv. Funct. Mater., 2009, 19, 479; (d) J. Zou, L. Liu, H. Chen, S. I. Khondaker, R. D. McCullough, Q. Huo and L. Zhai, Adv. Mater., 2008, 20, 2055; (e) F. Chen, B. Wang, Y. Chen and L.-J. Li, Nano Lett., 2007, 7, 3013; (f) A. Star, J. F. Stoddart, D. Steuerman, M. Diehl, A. Boukai, E. W. Wong, X. Yang, S.-W. Chung, H. Choi and J. R. Heath, Angew. Chem., Int. Ed., 2001, 40, 1721; (g) W. Z. Yuan, H. Zhao, X. Y. Shen, F. Mahtab, J. W. Y. Lam, J. Z. Sun and B. Z. Tang, Macromolecules, 2009, 42, 9400.

4 (a) I. O. Huyal, U. Koldemir, T. Ozel, H. V. Demir and D. Tuncel, J. Mater. Chem., 2008, 18, 3568.

5 (a) C. Wu, C. Szymanski and J. McNeill, Langmuir, 2006, 22, 2956; (b) D. Tuncel and H. V. Demir, Nanoscale, 2010, 2, 484; (c) I. O. Huyal, T. Ozel, D. Tuncel and H. V. Demir, Opt. Express, 2008, 16, 13391; (d) I. O. Ozel, T. Ozel, H. V. Demir and D. Tuncel, Opt. Express, 2010, 18, 670.

6 From this point on in the text, CNT abbreviation is used to denote multi-walled CNT.

7 (a) S. Fan, M. G. Chapline, N. R. Franklin, T. W. Tombler, A. M. Cassell and H. Dai, Science, 1999, 283, 512; (b) W. Z. Li, S. S. Xie, L. X. Qian, B. H. Chang, B. S. Zou, W. Y. Zhou, R. A. Zhao and G. Wang, Science, 1996, 274, 1701.

8 (a) T. Belin and F. Epron, Mater. Sci. Eng., B, 2005, 119, 105; (b) M. S. Dresselhaus, G. Dresselhaus, R. Saito and A. Jorio, Phys. Rep., 2005, 409, 47.

9 E. F. Antunes, A. O. Lobo, E. J. Corat, V. J. Trava-Airoldi, A. A. Martin and C. Verissimo, Carbon, 2006, 44, 2202.

10 A. S. Patole, S. P. Patole, J.-B. Yoo, Y.-H. Ahn and T.-H. Kim, J. Polym. Sci., Part A: Polym. Chem., 2009, 47, 1523.

11 Manuscript being prepared.

12 (a) B. C. Satishkumar, L. O. Brown, Y. Gao, C.-C. Wang, H.-L. Wang and S. K. Doorn, Nat. Nanotechnol., 2007, 2, 560; (b) K. Kostarelos, A. Bianco and M. Prato, Nat. Nanotechnol., $2009,4,627$. 